\title{
Preoperative Patient Education May Decrease Postoperative Opioid Use After Meniscectomy
}

\author{
Steven M. Andelman, M.D., Daniel Bu, B.S., Nicholas Debellis, M.D., \\ Chukwuma Nwachukwu, B.S., Nebiyu Osman, M.D., James N. Gladstone, M.D., and \\ Alexis C. Colvin, M.D.
}

\begin{abstract}
Purpose: To identify the current opioid prescribing and use practices after arthroscopic meniscectomy and to evaluate the role of preoperative patient education in decreasing postoperative opioid consumption. Methods: Patients undergoing arthroscopic meniscectomy were prospectively identified for inclusion. They were placed into 1 of 2 groups: Group 1 received no education regarding opioid use after surgery, whereas group 2 received a standardized overview on postoperative opioid use. Patients were assigned to the groups consecutively: Patients treated at the beginning of the study were assigned to group 1 , and patients treated at the end of the study were assigned to group 2. Data from group 1 were used to identify "normal" opioid prescribing and use practices and to guide patients in group 2 regarding normal postoperative opioid use. Patients were surveyed weekly for 4 weeks after surgery to determine the number of opioids taken. Postoperative opioid consumption was analyzed and compared between the 2 groups. Results: A total of 62 patients completed the study ( 32 in group 1 and 30 in group 2). Patients in group 1 were prescribed an average of 42.0 opioid pills (95\% confidence interval [CI], 34.0-51.0 pills) and used an average of 15.84 pills (95\% CI, 9.26-22.4 pills) after surgery, whereas patients in group 2 used an average of 4.00 pills (95\% CI, 2.12-5.88 pills) after surgery. Patients in group 2 used 11.84 fewer opioid pills $(P=.001)$, a $296 \%$ decrease in postoperative opioid consumption. The number of patients who continued to take opioid pills 4 weeks after surgery was 7 patients $(21.9 \%)$ in group 1 and 1 patient $(3.3 \%)$ in group 2 . Conclusions: Preoperative patient education regarding opioids may decrease postoperative opioid consumption and the duration for which patients take opioid pills after arthroscopic meniscectomy. Level of Evidence: Level II, prospective comparative study.
\end{abstract}

I n recent years, the rate of prescription opioid abuse and overdose in the United States has risen with such an alarming speed that the current state of affairs is frequently labeled the "opioid epidemic."1,2 As part of an effort to curb opioid abuse, physician prescribing practices have come under scrutiny. A national database study of opioid prescription patterns in the United States showed that orthopaedic surgeons are the thirdhighest prescribers of opioids, accounting for $7.7 \%$ of all opioid prescriptions in the country. ${ }^{3}$ Although

From the Department of Orthopaedic Surgery, SUNY Downstate Health Sciences University (S.M.A.), Brooklyn, New York; Department of Orthopaedic Surgery, Mount Sinai Hospital (D.B., N.D., C.N., J.N.G., A.C.C.), New York, New York; and Department of Orthopaedic Surgery and Sports Medicine, University of Connecticut (N.O.), Farmington, Connecticut, U.S.A.

Investigation performed at Mount Sinai Hospital.

The authors report the following potential conflicts of interest or sources of funding: J.N.G. receives personal fees from Arthrex, Mitek, Trice Medical, and Springer. Full ICMJE author disclosure forms are available for this article online, as supplementary material.

These data were previously presented as a podium presentation at the American Academy of Orthopaedic Surgeons Annual Meeting, Las Vegas, NV, orthopaedic surgeons provide a large number of opioid prescriptions, there is a paucity of literature to guide prescribers regarding the appropriate number of opioid pills to provide for various orthopaedic conditions.

Given the overwhelming data on opioid abuse, there has been interest in better detailing opioid prescription and use patterns. Within the orthopaedic literature, most research has focused on opioid prescribing and use practices after upper-extremity surgery, with reports noting that patients are prescribed 3 to 5 times the

March 12-16, 2019, and The American Orthopedic Society and Sports Medicine Annual Meeting, Boston, MA, June 12-16, 2019.

Received June 29, 2019; accepted October 8, 2019.

Address correspondence to Steven M. Andelman, M.D., Department of Orthopaedic Surgery, SUNY Downstate Health Sciences University, 450 Clarkson Ave, MSC 30,Brooklyn, NY 11203.E-mail: steve.andelman@gmail. com

(C) 2019 THE AUTHORS. Published by Elsevier Inc. on behalf of the Arthroscopy Association of North America. This is an open access article under the CC BY-NC-ND license (http://creativecommons.org/licenses/by-nc-nd/4.0/). 2666-061X/19804

https://doi.org/10.1016/j.asmr.2019.10.006 
number of opioid pills required. ${ }^{4-8}$ Considered on a national scale, this allows for an enormous number of unused opioid pills available for potential diversion for nontherapeutic use. ${ }^{8,9}$

These sobering findings have led some persons to advocate for the creation of patient- and procedurespecific guidelines to better approximate the appropriate number of opioid pills to prescribe after surgery. The goal of these guidelines is to provide appropriate analgesia while minimizing prescription of unnecessary opioid pills. Such guidelines advocate a multifaceted approach and stress the importance of patient education, provider education, identification of "normal" procedure-specific opioid consumption, and identification of factors associated with increased opioid use. ${ }^{10-12}$ Although there have been efforts both to identify patient risk factors for increased opioid use and to characterize the role of provider education to limit opioid prescriptions, there have been limited investigations regarding the role of formalized patient education to decrease postoperative opioid consumption. ${ }^{13-15}$

Arthroscopic knee meniscectomy is one of the most common procedures performed in orthopaedic surgery and is thus a frequent reason for prescription of postoperative opioids. ${ }^{16}$ The purposes of this study were to identify the current opioid prescribing and use practices after arthroscopic meniscectomy and to evaluate the role of preoperative patient education in decreasing postoperative opioid consumption. The hypothesis was that a lack of patient education regarding normal and appropriate postoperative opioid use would be a significant reason for increased opioid consumption after arthroscopic meniscectomy.

\section{Methods}

This analysis was set up as a prospective, nonrandomized, comparative, observational study. Subjects were identified prospectively through the clinic of 6 attending surgeons (A.C.C and J.N.G) at a tertiary referral institution. The inclusion criterion was any patient aged 18 years or older undergoing isolated arthroscopic unicompartmental meniscectomy. The exclusion criteria included a history of ipsilateral knee surgery, current opioid use, a documented allergy to opioids, preoperative opioid use, a self-reported history of opioid abuse, open meniscectomy, or meniscectomy performed in conjunction with other surgical procedures (chondral procedures, meniscal repair, anterior cruciate ligament reconstruction, and so on). Institutional review board approval was obtained prior to the study.

Patients were consecutively assigned to 2 separate cohorts prior to surgery. The goal of the first cohort (group 1) was to function as a pilot study to help illustrate current prescribing and use practices after arthroscopic meniscectomy. In this cohort, patients were given no instructions regarding postoperative opioid use and were instructed to take opioid pills as needed. Postsurgical opioid prescriptions were provided at the discretion of the attending surgeon, and prescribers were aware when a patient was enrolled in the study. To prevent bias, 20 prior arthroscopic meniscectomies were randomly selected for retrospective review, and the number of opioid pills prescribed was identified to ensure no change in prescribing practices after initiation of the study.

Patients were contacted weekly for 4 weeks after surgery. They were surveyed about the number of opioid pills they had taken in the previous week. They were also asked if they had been given any refills for further opioid medications by either the operative surgeon or any other prescriber during this time. Data from this cohort were analyzed to determine the average number of opioid pills prescribed and consumed after arthroscopic meniscectomy. Thus, data from the first cohort were used to determine normal opioid prescribing and use practices after arthroscopic meniscectomy.

After completion of data collection for group 1, all new eligible patients were assigned to the second cohort (group 2). The goal of the second cohort was to determine the role of preoperative education in reducing postoperative opioid consumption. Data from group 1 were used to guide patients regarding postoperative opioid use, and the reasoning behind this education process is detailed in the "Results" section. All patients in the second cohort received a prescription for 15 opioid pills (oxycodone/acetaminophen, 5/325 mg) and a standardized 3-minute overview by phone the night prior to surgery. This overview focused on appropriate postoperative use of opioids after arthroscopic meniscectomy and options for non-narcotic analgesia. The specific points discussed are listed in Table 1 .

Time was then given for patients to ask questions. After the phone conversation, a single-page standardized document reviewing all points discussed was emailed to the patient. A perioperative nurse also discussed these points with the patient on discharge. As in the first cohort, patients in the second cohort were contacted weekly for 4 weeks after surgery and queried regarding total opioids consumed in the past week.

Data were analyzed using SPSS software (version 25; IBM). Means were calculated with bias-corrected bootstrap methods, with 95\% confidence intervals (CIs). Paired $t$ tests with bias-corrected bootstrapping were used to test for significance in the number of opioids taken in consecutive weeks. The Mood median test and Fisher exact test were used to compare the median and total number of opioids consumed, respectively, between the 2 groups. A multivariate bias-corrected bootstrapped linear regression for the total number of opioids taken postoperatively was conducted using factors identified through initial univariate regression. Significance was set at $P<.05$. 
Table 1. Educational Points Discussed With Group 2

1. Opioids are for severe pain (VAS score of 8-10).

2. Nonsteroidal anti-inflammatory analgesics can be used for mild or moderate pain (VAS score of 1-7).

3. Cryotherapy is an effective means to decrease pain and swelling after surgery.

4. Most patients take $\leq$ opioid pills after surgery.

5. Most patients do not require opioids after the first postoperative week.

VAS, visual analog scale.

*Data in points 4 and 5 are based on data from group 1 and are further explained in the "Results" and "Discussion" sections.

\section{Results}

\section{Demographic Comparison of Groups 1 and 2}

A total of 62 patients completed the study: 32 in group 1 and 30 in group 2. Table 2 details the demographic statistics of the 2 cohorts. Comparison of the 2 groups is notable for statistically significant differences in the sex of the participants (more women in group 2), preoperative visual analog scale score (higher in group 2 ), and medical history of hypertension or hyperlipidemia (higher in group 1). The remainder of descriptive demographic statistics were equivalent between the 2 groups analyzed.

\section{Group 1: No Preoperative Education}

Patients in group 1 were prescribed an average of 42.0 opioid pills (95\% CI, 34.0-51.0 opioid pills). All pills prescribed were oxycodone/acetaminophen, 5/325 mg. Our review of prescriber practices revealed an average of 41.7 opioid pills prescribed after arthroscopic meniscectomy, showing no difference in prescribing practices after initiation of the study.

The mean value for postoperative opioid consumption was 15.84 pills (95\% CI, 9.26-22.4 pills), ranging from 0 to 57 pills, whereas the median number of opioids consumed was 7.5. Patients consumed the most opioid pills the first week after surgery (11.42), and opioid use decreased the following 3 weeks. Of the patients, $19(59.4 \%)$ took 10 or fewer total pills and 20 $(62.5 \%)$ stopped all opioid use after the first postoperative week. At 4 weeks after surgery, 7 patients $(21.9 \%)$ continued to take opioid pills. At the end of 4 weeks, there were 808 unused opioid pills.

\section{Group 2: Preoperative Education}

Data from group 1 were used to guide the education component used for group 2. First, the mean number of opioid pills consumed in group 1 (15.84) was used by the senior author (A.C.C.) to determine that all patients in group 2 would be prescribed 15 postoperative opioid pills. Second, the facts that most patients in group 1 took 10 or fewer total opioids after surgery $(59.4 \%)$ and most stopped opioid use 1 week after surgery $(62.5 \%)$ were used to guide patients regarding normal opioid use after arthroscopic meniscectomy during the education component discussed with group 2 .

The average number of opioid pills consumed after surgery was 4.00 (95\% CI, 2.12-5.88), and the median number of pills consumed was 2.0. Patients consumed the most opioid pills the first week after surgery (3.68; $95 \%$ CI, 2.93-4.75), and opioid consumption decreased in the following 3 weeks. Of the patients, $26(86.7 \%)$ used 10 or fewer opioids, $26(86.7 \%)$ stopped opioid use after the first postoperative week, and $1(3.3 \%)$ continued to use opioids at 4 weeks postoperatively. No patients requested a refill for further opioids.

\section{Comparison Between Group 1 and Group 2}

Patients in group 2, who received preoperative education regarding appropriate opioid use, consumed 11.84 fewer opioid pills $(P=.001)$ than patients in group 1 , who did not receive any education, representing a $296 \%$ decrease in postoperative opioid consumption. Figure 1 details the total and weekly opioid consumption of the 2 groups, and Table 3 illustrates differences in opioid use between the 2 groups. Patients in group 2 used fewer

Table 2. Patient Demographic Characteristics

\begin{tabular}{|c|c|c|c|}
\hline & Group 1 & Group 2 & $P$ Value \\
\hline$\overline{\text { Age, mean }(95 \% \mathrm{CI}) \text {, yr }}$ & $52.7(47.3-58.1)$ & $49.77(45.85-53.70)$ & .375 \\
\hline \multicolumn{4}{|l|}{ Sex, n $(\%)$} \\
\hline Female & $10(31.3)$ & $19(59.3)$ & .044 \\
\hline Preoperative pain level (VAS score, $0-10)$, mean $(95 \%$ CI) & $3.2(2.6-3.8)$ & $5.13(4.29-5.97)$ & $<.001$ \\
\hline \multicolumn{4}{|l|}{ Medical history, n (\%) } \\
\hline Depression & $5(15.6)$ & $5(16.1)$ & .957 \\
\hline Hyperlipidemia & $13(40.6)$ & $5(15.6)$ & .029 \\
\hline \multicolumn{4}{|l|}{ Insurance, $\mathrm{n}(\%)$} \\
\hline Commercial & $24(75)$ & $28(87.5)$ & \multirow[t]{2}{*}{.208} \\
\hline Medicare or Medicaid & $8(25)$ & $4(12.5)$ & \\
\hline No. of pills prescribed at discharge, mean $(95 \% \mathrm{CI})$ & $42.0(34.0-41.0)$ & $15.84(9.26-22.4)$ & $<.001$ \\
\hline
\end{tabular}

CI, confidence interval; VAS, visual analog scale. 
25

20

Group 1 - no education

1/. Group 2 - received education

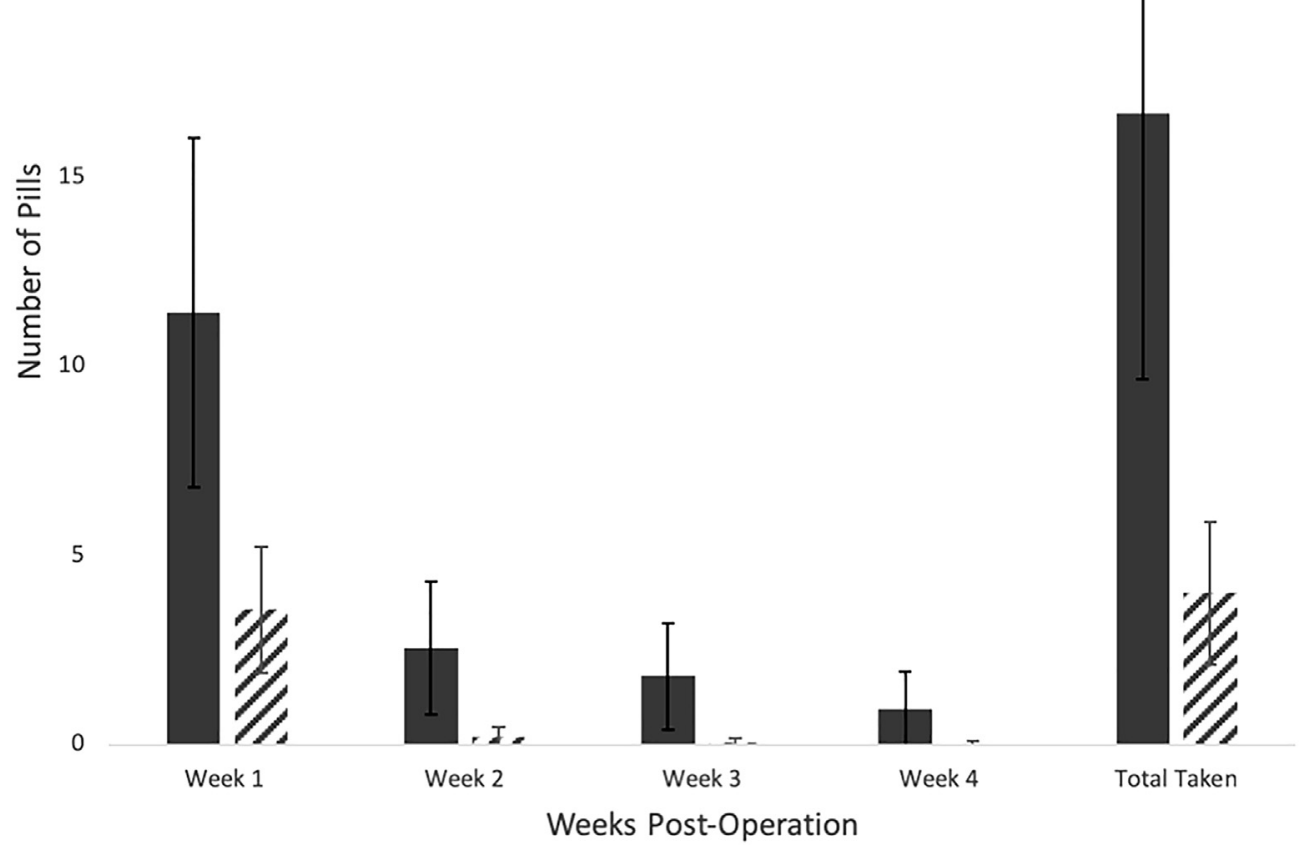

Fig 1. Comparison of opioid pills consumed between group 1 (no education, solid bars) and group 2 (preoperative education, hatched bars). The error bars represent 95\% confidence intervals. total opioids at each time point and used opioids for a shorter duration postoperatively. There were no variables identified via regression analysis to be associated with increased postoperative opioid use.

\section{Discussion}

This study shows that the initiation of a preoperative educational protocol regarding appropriate opioid use after arthroscopic meniscectomy can help decrease postoperative opioid consumption. We believe that there were 3 essential aspects that allowed for decreased opioid consumption in the group that received preoperative

Table 3. Trends in Opioid Consumption

\begin{tabular}{|c|c|c|c|}
\hline & $\begin{array}{l}\text { Group } 1 \\
(\mathrm{n}=32)\end{array}$ & $\begin{array}{l}\text { Group } 2 \\
(\mathrm{n}=30)\end{array}$ & $P$ Value \\
\hline $\begin{array}{l}\text { Average total opioids } \\
\text { consumed }\end{array}$ & 15.84 & 4.00 & .001 \\
\hline $\begin{array}{l}\text { Median total opioids } \\
\text { consumed }\end{array}$ & 7.5 & 2 & .02 \\
\hline \multicolumn{4}{|l|}{ Opioid consumption, n (\%) } \\
\hline $0-10$ pills & $19(59.4)$ & $26(86.7)$ & .02 \\
\hline 11-20 pills & $3(9.4)$ & $4(13.3)$ & .70 \\
\hline 21-30 pills & $2(6.3)$ & 0 & .49 \\
\hline$\geq 31$ pills & $7(21.9)$ & 0 & .01 \\
\hline $\begin{array}{l}\text { Patients using opioids at } 4 \mathrm{wk} \\
\text { postoperatively, n (\%) }\end{array}$ & $7(21.9)$ & $1(3.3)$ & .05 \\
\hline Total unused opioids & 808 & 330 & NA \\
\hline
\end{tabular}

education. First, these patients were given explicit information regarding appropriate use of opioid narcotics (for severe pain) in addition to non-narcotic analgesic options for mild and moderate pain (nonsteroidal antiinflammatory drugs [NSAIDs] and cryotherapy). This helped to prevent inappropriate use of opioids for mild and moderate pain when non-narcotic analgesics could otherwise be used. Second, on the basis of the results of the first cohort, patients were counseled that most patients took 10 or fewer pills after arthroscopic meniscectomy and that the majority of pills were taken within the first postoperative week. This helped to set expectations for patients regarding normal use practices and what to expect after surgery. Third, on the basis of the results of the first cohort, we provided a limited postoperative opioid prescription (15 pills), thus setting the expectation that large amounts of opioids are not required after this procedure. Taken together, these 3 elements emphasized that opioids are for severe pain, that they are generally only used in the first week after surgery, that most patients do not require more than 10 pills, and that there are effective non-narcotic options for analgesia.

Emphasis has been placed on characterizing opioid prescribing and use practices to help limit the number of postoperative opioid prescriptions. In the orthopaedic literature, much of this research has focused on upperextremity surgery. Kim et al. ${ }^{5}$ prospectively analyzed 
1,416 patients undergoing outpatient upper-extremity orthopaedic surgery such as carpal tunnel release, trigger finger release, and distal radius fracture open reduction-internal fixation over a 6-month period and reported that the average number of pills prescribed was 24 and the average number of pills used was 8.1, suggesting that surgeons were prescribing 3 times as many opioids as required. Likewise, Rodgers et al. ${ }^{6}$ retrospectively surveyed 250 patients 1 to 2 weeks after elective, outpatient soft-tissue upper-extremity surgery and reported that patients used on average 9 pills but were prescribed an average of 30 pills. They estimated that the 250 patients in their small study did not use 4,639 pills, which were thus available for diversion for nontherapeutic use. ${ }^{8}$ Looking specifically at carpal tunnel release, Chapman et al. ${ }^{4}$ reported that patients were prescribed an average of 21 pills and used an average of 4.3 pills, suggesting that patients were prescribed 5 times the number of opioid pills needed for this common procedure. These findings are especially concerning in light of a recent systematic review of 800 patients regarding opioid use practices after surgery that reported that $75 \%$ of patients did not properly store their opioids at home and $90 \%$ did not dispose of unused opioids in the correct fashion. ${ }^{17,18}$ Although much of the research on this topic has focused on outpatient upper-extremity surgery, the phenomenon of opioid over-prescription extends both to other aspects of orthopaedic surgery and to other specialties within medicine. ${ }^{19-24}$

In our first cohort, patients were prescribed nearly 3 times as many opioids as required. This finding is similar to results in the published literature that suggest that patients undergoing outpatient orthopaedic procedures are prescribed significantly more opioids than required for postoperative analgesia. To better approximate the number of opioids to give after surgery, some authors have tried to identify risk factors for increased postoperative opioid use. Female sex, younger age, surgical procedures involving manipulation of bony structures, high-energy fracture patterns, a history of depression and anxiety, a history of substance abuse, and current substance abuse have all been associated with increased opioid use after surgery. $4,5,7-9,14,15,22-24$

After analyzing our data from group 1, we hypothesized that a primary cause of increased opioid use after surgery was a lack of proper patient education regarding appropriate postoperative use. To our knowledge, this is the first study to evaluate the role of patient education as a means to decrease postoperative opioid use. We believed that the act of giving patients a prescription for postoperative opioids dictating that they "take 1 pill every 4 hours as needed for pain" could create significant confusion about when it is and is not appropriate to use narcotics. By explicitly stating that opioids are for severe pain only and then giving options for non-narcotic analgesia for mild and moderate pain, we helped to eliminate confusion about when it is appropriate to use narcotics in the postoperative setting. Perhaps more important, by using our data from group 1 regarding normal postoperative opioid use and providing a more limited opioid prescription ( 15 pills), we helped set the expectation for patients in group 2 that opioids are only to be used in the immediate postoperative period.

Recently, some groups have evaluated the role of NSAIDs alone for postoperative analgesia after arthroscopic meniscectomy. Pham et al. ${ }^{25}$ reported that, when given prescriptions for both an NSAID and an opioid after arthroscopic meniscectomy, more than half of patients reported taking the NSAID alone, and Daniels et al. ${ }^{26}$ reported $82 \%$ satisfactory postoperative analgesia when providing NSAIDs alone after arthroscopic meniscectomy. Our report helps build on these findings, showing that, in those situations in which either surgeon or patient preference dictates the prescription of a postoperative opioid, proper preoperative education can help to limit the number of opioid pills taken in the postoperative period.

In summary, there are 2 conclusions that can inferred from this study. First, as has been shown in similar previous studies, there is a trend toward over-prescription of opioids after arthroscopic meniscectomy, and second, patient education regarding proper opioid use may help substantially decrease the number of opioids taken after arthroscopic meniscectomy. Although this study pertains only to arthroscopic meniscectomy, we believe that the use of patient education to decrease postoperative opioid demand could be extrapolated to other surgical procedures both in orthopaedic surgery and within other surgical specialties.

\section{Limitations}

There are several limitations to this study that are important to recognize. First, this study involved a small sample size, and a power analysis was not performed, making a type II error more likely. Second, the type of meniscal tear and degree of associated chondromalacia was not recorded or part of the analysis. This information could have an effect on postoperative opioid consumption that would not be recognized in our analysis. Third, we did not control for perioperative analgesic techniques regarding both standardization of anesthesia and surgeon use of perioperative injections. However, although this was not part of the analysis, surgeons were asked not to alter any perioperative analgesic techniques for the duration of the study. Fourth, although patients were given recommendations regarding postoperative use of cryotherapy and NSAIDs, they were not given explicit prescriptions for standardized cryotherapy units or specific NSAIDs; thus, decisions regarding the use of these modalities (e.g., which type of NSAID to use) was left up to the patient. As such, it may be that use of a specific NSAID 
in a specific manner could affect postoperative opioid consumption and that such an effect would not be captured by our analysis. Fifth, there was a risk of reporter bias because patients were at risk of underreporting the number of opioids taken given the current negative connotations associated with opioid use.

Last, and perhaps most important, this study functioned as an analysis of 2 consecutive, nonblinded, prospective cohorts. Although this recruitment method was purposeful because it allowed for characterization of baseline normal postoperative opioid use after arthroscopic meniscectomy to help establish guidelines for opioid use in group 2, a concurrent randomized allocation of patients would have better eliminated the risk of bias.

\section{Conclusions}

Preoperative patient education regarding opioids may decrease postoperative opioid consumption and the duration for which patients take opioid pills after arthroscopic meniscectomy.

\section{References}

1. Guy GP Jr, Zhang K, Bohm MK, et al. Vital signs: Changes in opioid prescribing in the United States, 2006-2015. MMWR Morb Mortal Wkly Rep 2017;66:697-704.

2. Rudd RA, Seth P, David F, Scholl L. Increases in drug and opioid-involved overdose deaths-United States, 20102015. MMWR Morb Mortal Wkly Rep 2016;65:1445-1452.

3. Volkow ND, McLellan TA, Cotto JH, Karithanom M, Weiss SR. Characteristics of opioid prescriptions in 2009. JAMA 2011;305:1299-1301.

4. Chapman T, Kim N, Maltenfort M, Ilyas AM. Prospective evaluation of opioid consumption following carpal tunnel release surgery. Hand (N Y) 2017;12:39-42.

5. Kim N, Matzon JL, Abboudi J, et al. A prospective evaluation of opioid utilization after upper-extremity surgical procedures: Identifying consumption patterns and determining prescribing guidelines. J Bone Joint Surg Am 2016;98:e89.

6. Rodgers J, Cunningham K, Fitzgerald K, Finnerty E. Opioid consumption following outpatient upper extremity surgery. J Hand Surg Am 2012;37:645-650.

7. Cheatle MD. Prescription opioid misuse, abuse, morbidity, and mortality: Balancing effective pain management and safety. Pain Med 2015;16:S3-S8 (suppl 1).

8. Devin CJ, Lee DS, Armaghani SJ, et al. Approach to pain management in chronic opioid users undergoing orthopaedic surgery. J Am Acad Orthop Surg 2014;22:614-622.

9. Manchikanti L, Kaye AM, Knezevic NN, et al. Responsible, safe, and effective prescription of opioids for chronic noncancer pain: American Society of Interventional Pain Physicians (ASIPP) guidelines. Pain Physician 2017;20:S3-S92.

10. Soffin EM, Waldman SA, Stack RJ, Liguori GA. An evidencebased approach to the prescription opioid epidemic in orthopedic surgery. Anesth Analg 2017;125:1704-1713.
11. Stanek JJ, Renslow MA, Kalliainen LK. The effect of an educational program on opioid prescription patterns in hand surgery: A quality improvement program. J Hand Surg Am 2015;40:341-346.

12. Johnson SP, Chung KC, Zhong L, et al. Risk of prolonged opioid use among opioid-naïve patients following common hand surgery procedures. J Hand Surg Am 2016;41: 947-957.e3.

13. Webster LR. Risk factors for opioid-use disorder and overdose. Anesth Analg 2017;125:1741-1748.

14. Seth P, Scholl L, Rudd RA, Bacon S. Overdose deaths involving opioids, cocaine, and psychostimulants-United States, 2015-2016. MMWR Morb Mortal Wkly Rep 2018;67: 349-358.

15. Centers for Disease Control and Prevention. Vital signs: Overdoses of prescription opioid pain relievers-United States, 1999-2008. MMWR Morb Mortal Wkly Rep 2011;60: 1487-1492.

16. Cullen KA, Hall MJ, Golosinskiy A. Ambulatory surgery in the United States, 2006. Natl Health Stat Report 2009:1-25.

17. Bicket MC, Long JJ, Pronovost PJ, Alexander GC, Wu CL. Prescription opioid analgesics commonly unused after surgery: A systematic review. JAMA Surg 2017;152:1066-1071.

18. Bartels K, Mayes LM, Dingmann C, Bullard KJ, Hopfer CJ, Binswanger IA. Opioid use and storage patterns by patients after hospital discharge following surgery. PLoS One 2016;11:e0147972.

19. Finger A, Teunis T, Hageman MG, Ziady ER, Ring D, Heng M. Association between opioid intake and disability after surgical management of ankle fractures. J Am Acad Orthop Surg 2017;25:519-526.

20. Gauger EM, Gauger EJ, Desai MJ, Lee DH. Opioid use after upper extremity surgery. J Hand Surg Am 2018;43: 470-479.

21. Kumar K, Gulotta LV, Dines JS, et al. Unused opioid pills after outpatient shoulder surgeries given current perioperative prescribing habits. Am J Sports Med 2017;45:636-641.

22. Miller A, Kim N, Ilyas AM. Prospective evaluation of opioid consumption following hand surgery performed wide awake versus with sedation. Hand (N Y) 2017;12:606-609.

23. O'Neil JT, Wang ML, Kim N, Maltenfort M, Ilyas AM. Prospective evaluation of opioid consumption after distal radius fracture repair surgery. Am J Orthop (Belle Mead NJ) 2017;46:E35-E40.

24. Waljee JF, Zhong L, Hou H, Sears E, Brummett C, Chung KC. The use of opioid analgesics following common upper extremity surgical procedures: A national, population-based study. Plast Reconstr Surg 2016;137:355e-364e.

25. Pham H, Pickell M, Yagnatovsky M, et al. The utility of oral nonsteroidal anti-inflammatory drugs compared with standard opioids following arthroscopic meniscectomy: A prospective observational study. Arthroscopy 2019;35: 864-870.el.

26. Daniels SD, Garvey KD, Collins JE, Matzkin EG. Patient satisfaction with nonopioid pain management following arthroscopic partial meniscectomy and/or chondroplasty. Arthroscopy 2019;35:1641-1647. 